\title{
ANÁLISE TERMODINÂMICA DO PROCESSO DE REFORMA DO METANO COM DIÓXIDO DE CARBONO
}

\author{
CARLOS P. S. ROCHA ${ }^{1}$, JOSÉ A. PACÍFICO ${ }^{1}$, EDILSON de JESUS ${ }^{1}$, ANTÔNIO S. SILVA² e \\ SAMIA T. MACIEL ${ }^{1}$ \\ ${ }^{1}$ Universidade Federal de Sergipe, Departamento de Engenharia Química \\ ${ }^{2}$ Universidade Federal de Sergipe, Departamento de Matemática \\ E-mail para contato: pheliperocha16@hotmail.com
}

RESUMO - Atualmente, tanto no cenário nacional bem como no cenário mundial são notáveis as reservas petróleo e gás natural. Segundo a Petrobras, milhares de litros de óleo bruto e gás natural são extraídos e processados diariamente dando origem a diversos produtos, tais como: gás de síntese, parafinas, gasolina natural e combustíveis líquidos através da tecnologia GTL (Gas to Liquid). Neste contexto, a análise termodinâmica do processo de reforma do metano com dióxido de carbono foi analisada tendo em vista a sua otimização empregando a minimização da energia de Gibbs. Deste modo, o presente estudo têve como objetivo a minimização dos custo de produção do processamento da reforma do $\mathrm{CH} 4-\mathrm{CO} 2$ realizada em reator de leito fixo. Os resultados mostraram que temperatura de 1029,21 K seria a mínima para operação do processo, para evitar a formação de coque, o que evitaria a desativação do catalisador pela deposição do carbono. Tal resultado mostraram que pode-se operar o processo em temperatura menor que a recomendada na literatura por vários autores, de 1073,15 K, como mostrou Serrano-Lotina e Daza (2014), o que possibilita reduzir os custos energéticos do processo.

\section{INTRODUÇÃO}

A indústria petrolífera tem papel importante na sociedade atual, centenas de produtos são feitos a partir de derivados do petróleo, que é um óleo de origem fóssil, que levou milhões de anos para ser formado nas rochas sedimentares e se tornou a principal fonte de energia do mundo moderno. No Brasil, a maior parte das reservas está nos campos marítimos, em lâminas d'água com profundidades maiores do que as dos demais países produtores. Nas refinarias, o óleo bruto passa por uma série de processos até a obtenção dos produtos derivados, como gasolina, diesel, lubrificantes, nafta, querosene de aviação. A presença dos produtos feitos a partir de derivados do petróleo na sociedade é notável, batom, chiclete, plástico, tintas, resinas e velas contêm derivados de petróleo (PETROBRAS, 2014).

No contexto da indústria petrolífera, pode-se destacar a importância do gás natural, obtido na exploração do petróleo, sendo usado como ingrediente importante na geração de energia. No entanto, o gás natural é também matéria prima dominante para a produção de vários produtos químicos, sendo 


\section{9 a 22 de outubro de 2014 \\ Florianópolis/SC}

antes convertido a gás de síntese, ou syngas como é conhecido industrialmente, são exemplos de derivados do gás natural a amônia, o metanol e o dimetiléter (DME), além de outros combustíveis líquidos obtidos através da síntese de Fischer-Tropsch.

O gás de síntese, ou syngas como é conhecido internacionalmente, é uma mistura composto por monóxido de carbono (CO) e gás hidrogênio $\left(\mathrm{H}_{2}\right)$ (MIRVAKILI et al., 2014). O gás de síntese pode ser produzido a partir do carvão, do coque de petróleo, da biomassa, e do gás natural através do processo de reforma do metano. A produção de gás de síntese a partir de gás natural é uma das tecnologias mais importantes na indústria, e é um assunto atraente e desafiante para a utilização de metano e dióxido de carbono (VASCONCELOS, 2006; PETERSEN e AASBERG, 2011; YAO et al., 2013; ZANGANEH, REZAEI e ZAMANIYAN, 2013).

A reforma seca é o processo no qual o metano reage com o dióxido de carbono para formar o gás de síntese, como mostra a Equação 1. Este processo apresenta algumas vantagens, tais como uma razão $\mathrm{H}_{2} / \mathrm{CO}$ inferior (igual a 1), se comparado com outros processos de reforma.

$$
\mathrm{CH}_{4}+\mathrm{CO}_{2} \rightleftarrows 2 \mathrm{CO}+2 \mathrm{H}_{2} \quad \Delta \mathrm{H}_{298}^{0}=247 \mathrm{~kJ} / \mathrm{mol}
$$

No processo ocorre ainda a reação reversa de Water-Gas Shift, representada pela Equação 2, promovendo a formação de água e mais monóxido de carbono (CHAO et al., 2012).

$$
\mathrm{CO}_{2}+\mathrm{H}_{2} \rightleftarrows \mathrm{CO}+\mathrm{H}_{2} \mathrm{O} \quad \Delta \mathrm{H}_{298}^{0}=41,0 \mathrm{~kJ} / \mathrm{mol}
$$

Sua principal desvantagem é a rápida desativação de catalisadores de níquel como resultado da deposição de carbono, na forma de coque, que é formado devido a reações paralelas indesejáveis, resultante da reação de Boudouard, expressa pela Equação 3, da decomposição direta do metano, expressa pela Equação 4 (ZANGANEH, REZAEI e ZAMANIYAN, 2013).

$$
\begin{array}{ll}
2 \mathrm{CO} \rightleftarrows \mathrm{C}+\mathrm{CO}_{2} & \Delta H_{298}^{0}=-172,4 \mathrm{~kJ} / \mathrm{mol} \\
\mathrm{CH}_{4} \rightleftarrows \mathrm{C}+2 \mathrm{H}_{2} & \Delta H_{298}^{0}=74,9 \mathrm{~kJ} / \mathrm{mol}
\end{array}
$$

Neste trabalho, o processo de interesse é o de reforma seca que utiliza dióxido de carbono como reagente, junto com o metano. A análise termodinâmica consiste em simular condições operacionais, para verificar as melhores condições de temperatura, pressão e composição da alimentação. As condições de realização do processo devem ser tais que haja a mínima formação de coque possível, sem no entanto sacrificar o rendimento da reforma. Para tanto, deve-se conhecer como o sistema se comporta no equilíbrio. Então, será a análise deste estado que permitirá determinar as condições ótimas de operação, fazendo-se necessário o cálculo das constantes de equilíbrio e outros parâmetros, para ser possível o cálculo da energia de Gibbs do sistema na condição de mínimo.

O presente trabalho está organizado da seguinte forma: a seção 2 apresenta a metodologia utilizada no trabalho, a seção 3 exibe os resultados obtidos, a seção 4 apresenta a discussão dos resultados obtidos e finalmente a seção 5 apresenta a conclusão do trabalho. 


\section{METODOLOGIA}

Neste trabalho, as reações que representam o sistema reacional são as reações: de decomposição catalítica do metano; reversa de Water-Gas shift e reversa de Boudouard, chamadas aqui, respectivamente, de reações 1,2 e 3 .

\subsection{Cálculo das constantes de equilíbrio}

Neste trabalho, as constantes de equilíbrio foram calculadas com o método da minimização da energia Gibbs. Sendo a fase gasosa assumida como um gás ideal, a energia livre total do sistema pode ser expressa como (NEMATOLLAHI et al., 2012):

$G=\sum_{i} n_{i} G_{i}^{0}+R T \sum_{i} n_{i} \ln \left(y_{i} P\right)$

onde: $\mathrm{G}$ é a energia livre total do sistema, em $\mathrm{J} ; n_{i}$ é o número de moles da espécie $i$, em mol; $G_{i}^{0}$ é a energia molar de Gibbs padrão de formação da espécie $i$, em $\mathrm{J} / \mathrm{mol}$; R é a constante universal do gases ideais, em $\mathrm{J} /(\mathrm{mol} \mathrm{K})$; T é a temperatura do sistema, em $\mathrm{K} ; y_{i}$ é a fração molar da espécie $i$ e $\mathrm{P}$ é a pressão do sistema, em atm.

Pacífico (2009) mostrou que de maneira geral a solução da Equação 5 é a Equação 6 que resulta na expressão da constante de equilíbrio, Equação 7. A partir destes resultados, ainda de acordo com Pacífico (2009) pode-se obter a Equação 8, derivada da equação de Vant't Hoff. A variação de entalpia é dada pela Equação 9. Então, substituindo a Equação 10 na Equação 9 e aplicando tal resultado na Equação 8, obtém-se a Equação 11, que é a expressão final que fornece as constantes de equilíbrio para o sistema reacional.

$$
\begin{aligned}
& -\frac{\sum_{i} v_{i} G_{i}}{R T}=\sum_{i} y_{i}^{v_{i}}=\ln \left(\prod_{i} y_{i}^{v_{i}}\right) \\
& K_{i}(T)=\exp \left(-\frac{\Delta G_{i}^{0}}{R T}\right) \\
& \ln K_{i}=\ln \left(\frac{-\Delta G^{\circ}}{R T}\right)+\int_{T r}^{T} \frac{\Delta H_{i}^{\circ}(T)}{R T^{2}} d T \\
& \Delta H_{i}^{\circ}(T)=\Delta H_{i}^{\circ}(T r)+\int_{T r}^{T} C_{p, i}^{\circ}(T) d T \\
& C_{p, i}^{\circ}(T)=A_{i}+B_{i} T+C_{i} T^{2}+D_{i} T^{3}+E_{i} T^{-2} \\
& \ln _{i}=\ln \left(\frac{-\Delta G^{\circ}}{R T r}\right)+\frac{A_{i}}{R} \ln \left(\frac{T}{T r}\right)+\frac{B_{i}}{2 R}(T-T r)+\frac{C_{i}}{6 R}\left(T^{2}-\operatorname{Tr}^{2}\right)+\frac{D_{i}}{12 R}\left(T^{3}-\operatorname{Tr}^{3}\right)+\frac{E_{i}}{2 R}\left(T^{-2}-\right. \\
& \left.\operatorname{Tr}^{-2}\right)+\frac{1}{R}\left[-\Delta H_{i}^{\circ}(\operatorname{Tr})+A_{i} T r+\frac{B_{i}}{2} \operatorname{Tr}^{2}+\frac{C_{i}}{3} \operatorname{Tr}^{3}+\frac{D_{i}}{4} \operatorname{Tr}^{4}-\frac{E_{i}}{T r}\right]\left(\frac{1}{T}-\frac{1}{T r}\right)
\end{aligned}
$$

onde: $v_{i}$ é o coeficiente estequiométrico da espécie $i ; G_{i}$ é a energia de Gibbs da espécie $i ; K_{i}$ é a 
constante de equilíbrio da espécie $i ; \Delta G_{i}^{0}$ é a variação da energia livre de Gibbs no estado padrão; $T r$ é a temperatura de referência; $\Delta H^{\circ}{ }_{i}$ é a variação de entalpia; $C_{p, i}^{\circ}(T)$ é a capacidade calorífica da espécie $i ; A_{i}, B_{i}, C_{i}, D_{i}$ e $E_{i}$ são parâmetros específicos da espécie $i$ e foram obtidos da literatura exposta por Reid, Prausnitz e Poling (1997).

\subsection{Determinação das composições no equilíbrio}

Com base no sistema reacional, que também contém o gás argônio como inerte, foi realizado o balanço de massa para as espécies envolvidas no equilíbrio, conforme mostra a Tabela 1 , onde $x_{1}, x_{2}$ e $x_{3}$ são, respectivamente, os graus de avanço das reações 1,2 e 3 .

Tabela 1 - Balanços de massa para as espécies envolvidas

\begin{tabular}{cccc}
\hline Espécies & $\begin{array}{c}\text { Número de moles } \\
\text { iniciais }\end{array}$ & $\begin{array}{c}\text { Números de moles } \\
\text { finais }\end{array}$ & $\begin{array}{c}\text { Frações molares no } \\
\text { equilíbrio }\end{array}$ \\
\hline $\mathrm{CH}_{4}$ & 0,10 & $0,10-x_{1}$ & $\frac{0,10-x_{1}}{1+x_{1}+x_{3}}$ \\
$\mathrm{CO}_{2}$ & 0,15 & $0,15-x_{2}-x_{3}$ & $\frac{0,15-x_{2}-x_{3}}{1+x_{1}+x_{3}}$ \\
$\mathrm{CO}$ & 0 & $x_{2}+2 x_{3}$ & $\frac{x_{2}+2 x_{3}}{1+x_{1}+x_{3}}$ \\
$\mathrm{H}_{2}$ & 0 & $2 x_{1}-x_{2}$ & $\frac{2 x_{1}-x_{2}}{1+x_{1}+x_{3}}$ \\
$\mathrm{H}_{2} \mathrm{O}$ & 0 & $x_{2}$ & $\frac{x_{2}}{1+x_{1}+x_{3}}$ \\
$\mathrm{Ar}$ & 0,75 & 0,75 & 0,75 \\
\hline Total & 1 & $1+x_{1}+x_{3}$ & $1+x_{1}+x_{3}$ \\
\hline
\end{tabular}

Além disso, no modelo foram adicionadas as restrições 1 e 2 que determinam, respectivamente que: não será consumido mais metano que o especificado, como mostra a Equação 12; a reação reversa de Water-Gas Shift não terá mais hidrogênio molecular que o que será produzido pela decomposição catalítica do metano, como mostra a Equação 13.

$0<x_{1}<0,1$

$0<x_{2}<2 x_{1}$

onde $x_{1}$ e $x_{2}$ são, respectivamente os graus de avanço das reações 1 e 2 . 
A partir das composições no equilíbrio pode-se escrever as seguintes expressões, derivadas da definição das constantes de equilíbrio:

$K_{1}=\frac{\left(2 x_{1}-x_{2}\right)^{2}}{\left(0,10-x_{1}\right)\left(1+x_{1}+x_{3}\right)}$

$K_{2}=\frac{x_{2}\left(x_{2}+2 x_{3}\right)}{\left(0,15-x_{2}-x_{3}\right)\left(2 x_{1}-x_{2}\right)}$

$K_{3}=\frac{\left(x_{2}+2 x_{3}\right)^{2}}{\left(0,15-x_{2}-x_{3}\right)\left(1+x_{1}+x_{3}\right)}$

onde: $K_{1}, K_{2}$ e $K_{3}$ são, respectivamente, as constantes de equilíbrio das reações: de decomposição catalítica do metano; reversa de Water-Gas shift e reversa de Boudouard.

Substituindo a expressão das respectivas constantes de equilíbrio, segundo a Equação 11, obtém-se o sistema de equações algébricas acopladas não lineares que possibilita o cálculo das composições de equilíbrio. A solução do sistema foi realizada através do software MatLab.

\subsection{Metodologia experimental para validação do modelo}

Foi utilizado micro-reator de quartzo, com dimensões de $50 \mathrm{~cm}$ de comprimento por $15 \mathrm{~mm}$ de diâmetro interno, em presença do catalisador de $\mathrm{Ni}(5,11 \% \mathrm{wt}) / \gamma$-Al2O3. Operou-se o sistema sob condições de pressão constante de 1.0 bar, razão de alimentação dos reagentes (mol $/ \mathrm{mol}$ ) $\left(\mathrm{CH}_{4}: \mathrm{CO}_{2}: \mathrm{Ar}=2: 3: 15\right)$, temperatura variando entre $773,15 \mathrm{~K}$ até $1148,15 \mathrm{~K}$ com incremento de 25 $\mathrm{K}$ em $25 \mathrm{~K}$, taxa de aquecimento de $5 \mathrm{~K} / \mathrm{min}$ e duração de 120 minutos de reação em cada intervalo de temperatura.

O catalisador de níquel suportado em gama alumina empregado nesta avaliação foi preparado pela dissolução do sal precursor de $\mathrm{Ni}\left(\mathrm{NO}_{3}\right)_{2} \cdot 6 \mathrm{H}_{2} \mathrm{O}$ (Sigma-Aldrich) 2,5M em água destilada e impregnado durante 6 horas em temperatura de 353K. Após a etapa de impregnação o catalisador foi calcinado e reduzido na temperatura de $973 \mathrm{~K}$ durante 6 horas com fluxo contínuo de $60 \mathrm{~cm}^{3} / \mathrm{min}$ de argônio e hidrogênio puro, respectivamente. As avaliações foram realizadas empregando-se $1,00 \mathrm{~g}$ de catalisador, alimentação com vazão volumétrica constante de 400 $\mathrm{cm}^{3} /$ min e tempo de contato de $\left(\mathrm{W} / \mathrm{FTi}=0,15 \mathrm{~g}_{\text {cat }} \mathrm{s} \mathrm{cm}^{-3}\right)$, GHSV $=2400 \mathrm{~cm}^{3} \mathrm{gcat}^{-1} \mathrm{~h}^{-1}$. Os resultados foram analisados via on-line na alimentação e produtos através de cromatógrafo a gás (GC Varian 3800) equipado com detector de condutividade térmica (TCD) e coluna de aço inoxidável empacotada de 6 pés por 1/8 de polegada (Alltech, carbosphere 60/80). O sistema em operação está representado na Figura 1. 


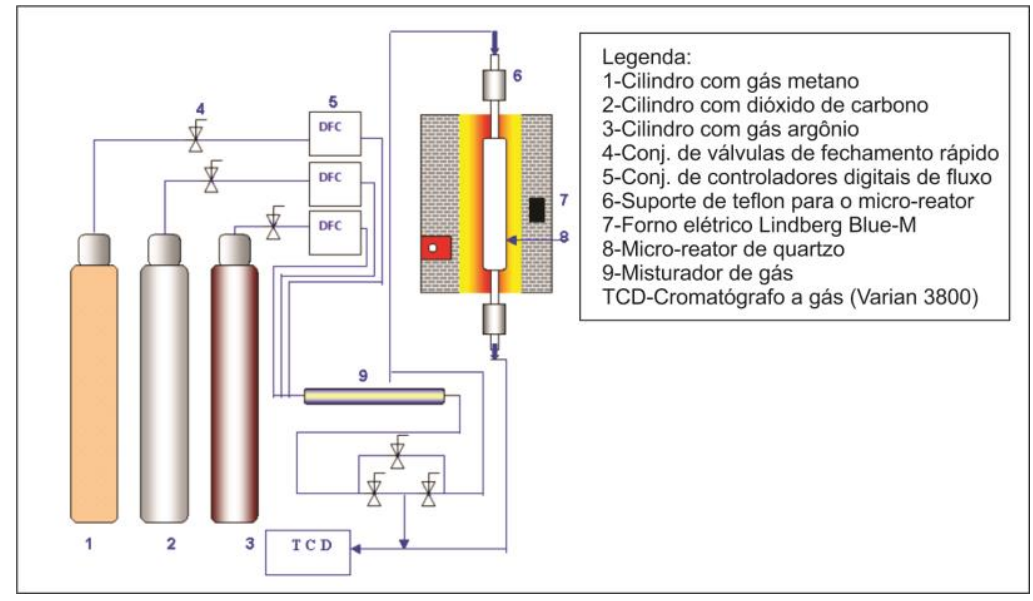

Figura 1 - Esquema do aparato experimental

O forno está equipado com um indicador-controlador linear de temperatura. Um segundo termopar está introduzido no interior do micro reator, indicando medidas no interior do reator.

\section{RESULTADOS E DISCUSSÃO}

Através da simulação realizada, foi verificado que a mínima temperatura teórica de operação que evita a formação de coque é de $1029,21 \mathrm{~K}$, pois nesta situação todo o carbono livre gerado pela reação de decomposição do metano foi consumido pela reação reversa de Boudouard. Tal resultado mostrou que pode-se operar o processo de reforma do metano com $\mathrm{CO}_{2}$ em temperatura menor que a recomendada na literatura, isto é , 1073,15 K (SERRANO-LOTINA e DAZA, 2014). Por esta razão, o estudo da análise termodinâmica demonstra que é possível a realização da reação de reforma $\mathrm{CH}_{4} / \mathrm{CO}_{2}$ em temperaturas menores que $1073,15 \mathrm{~K}$, o que é ótimo para a realização desta reforma em termos industrias, o que significa redução de custos operacionais e de energia. A Figura 2 exibe o gráfico da variação das constantes de equilíbrio em função da temperatura.

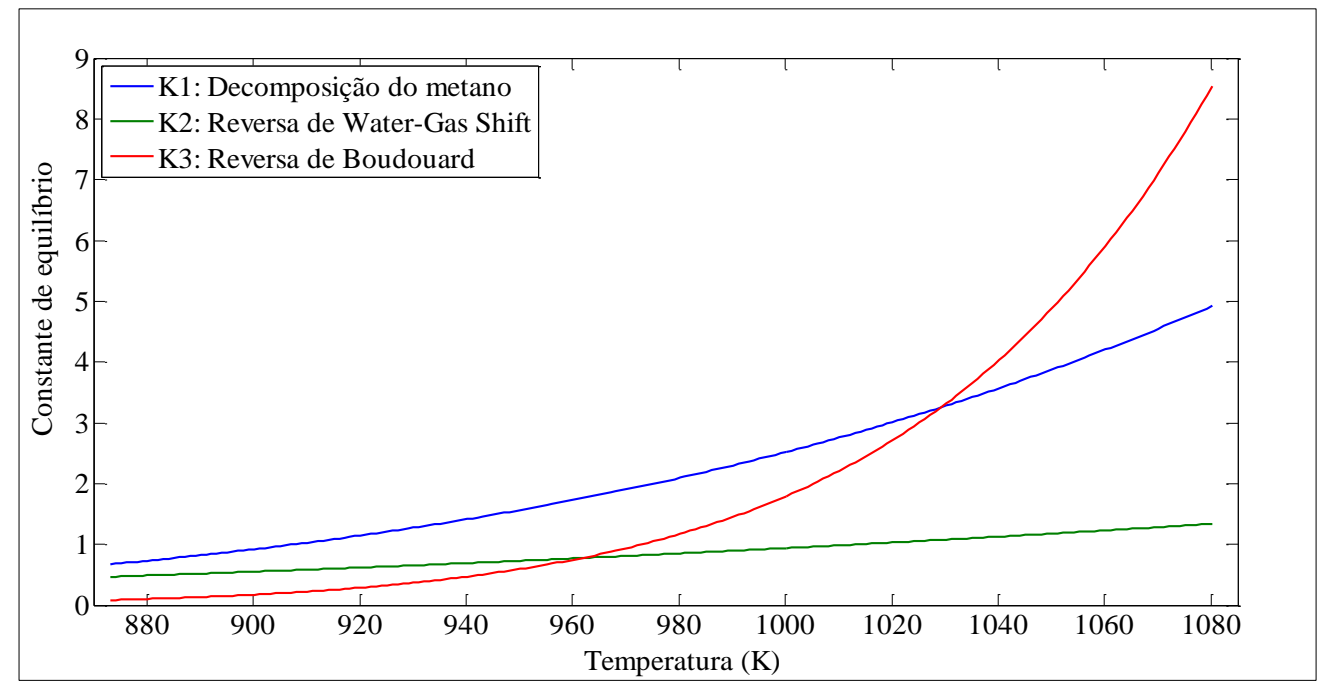

Figura 2 - Gráfico da variação das constantes de equilíbrio em função da temperatura 
As composições dos testes experimentais foram confrontadas com os resultados de simulação praticados na temperatura de $1029,21 \mathrm{~K}$ no equilíbrio químico, as quais atingiram os patamares da ordem de $55 \%$ de monóxido de carbono; 37,12 \% de hidrogênio; 3,6 \% de dióxido de carbono; 2,61 $\%$ de água e 1,64 \% de metano. A Figura 3 mostra o gráfico das frações molares estimadas e experimentais.

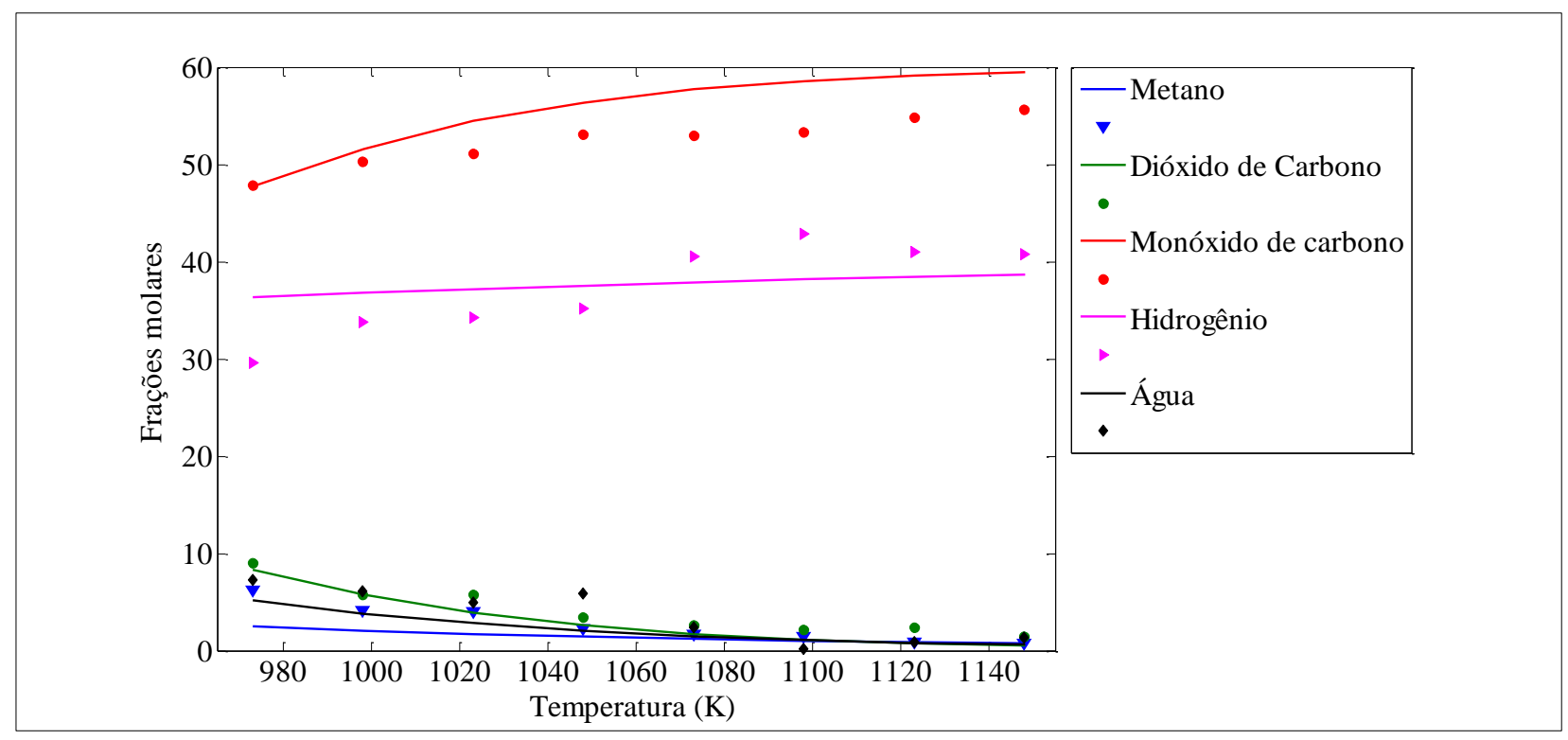

Figura 3 - Gráfico das frações molares estimadas. representadas pelas linhas, e experimentais, representadas pelos pontos

Apesar dos resultados teóricos obtidos, ainda deve-se levar em conta que o processo ocorre com a presença de catalisador e, portanto, a temperatura de sinterização do mesmo deve ser considerada, pois caso esta seja abaixo de 1029,21 K ela que será a máxima temperatura que poderá ser usada no processo, o que inevitavelmente permitiria a formação de coque, que pode ser detectado pelo simples balanço de massa do carbono. Tal temperatura reduziria a conversão da reação. Portanto, uma análise operacional deve ser realizada para verificar o que seria mais lucrativo em termos da temperatura de operação, para verificar se é vantajoso permitir que ocorra a sinterização de parte do catalisador para se obter maiores conversões.

\section{CONCLUSÃO}

A importância dos processos de reforma industrialmente é clara. Centenas de produtos são produzidos a partir de compostos obtidos direta ou indiretamente por um processo de reforma. Neste trabalho foi realizada a análise termodinâmica do processo de reforma seca, o que permitiu mostra que, teoricamente, a temperatura de $1029,21 \mathrm{~K}$ seria a mínima para operação do processo, pois todo o coque produzido seria consumido pela reação de Boudouard, o que evitaria a desativação do catalisador pela deposição do carbono. Tal resultado mostra que pode-se operar o processo em temperatura menor que a recomendada na literatura por vários autores, de 1073,15 K, como mostrou Serrano-Lotina e Daza (2014), o que possibilita reduzir os custos energéticos do processo. 


\section{REFERÊNCIAS}

CHAO, Zhongxi; WANG, Yuefa; JAKOBSEN, Jana P.; FERNANDINO, Maria; JAKOBSEN, Hugo A. Numerical investigation of the sorption enhanced steam methane reforming in a fluidized bed reactor. Energy Procedia. v. 26, p. 15 - 21, 2012.

MIRVAKILI, A; HERAVI, M; KARIMIPOURFARD, D; RAHIMPOUR, M.R; Simultaneous synthesis gas and styrene production in the optimized thermally coupled reactor. Journal of Natural Gas Science and Engineering. v. 16, p. 18-30, 2014.

NEMATOLLAHI, Behzad; REZAEI, Mehran; Lay, EBRAHIM Nemati; KHAJENOORI, Majid. Thermodynamic analysis of combined reforming process using Gibbs energy minimization method: In view of solid carbon formation. Journal of Natural Gas Chemistry. v. 21, p. 694-702, 2012.

PETERSEN, K. AASBERG- et al. Natural gas to synthesis gas - Catalysts and catalytic processes. Journal of Natural Gas Science and Engineering. v. 3, p. 423-459, 2011.

PETROBRAS. Petróleo. Disponível em: http://www.petrobras.com.br/pt/energia-e-tecnologia/fontesde-energia/petroleo/. Acesso em: 20 de jan. 2014.

REID, Robert C.; PRAUSNITZ, John M.; POLING, Bruce E. The properties of gases and liquids. 4. ed. New York: McGraw-Hill, 1997. 741 p.

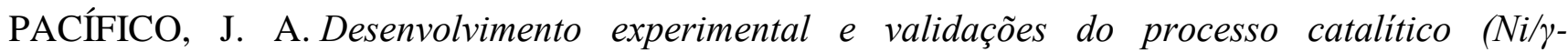
$\mathrm{Al}_{2} \mathrm{O}_{3} / \mathrm{CeO}_{2}-\mathrm{Al}_{2} \mathrm{O}_{3}$ ) de reforma metano-dióxido de carbono em reator de leito fluidizado. Recife, 2009. 242 p. Dissertação (Doutorado em Engenharia Química) - Universidade Federal de Pernambuco, Recife, 2009. [Orientador: Prof. Dr. César Augusto M. de Abreu].

SERRANO-LOTINA, A; DAZA, L. Influence of the operating parameters over dry reforming of methane to syngas. International Journal of Hydrogen Energy. v.39, p. 4089-4094, 2014.

VASCONCELOS, Nice de. Reforma a vapor do metano em catalisadores à base de níquel promovidos com nióbia. 2006. 94p. Dissertação (Mestrado em Engenharia Química) - Universidade Federal Fluminense, Niterói, 2006.

YAO, Lu et al. Comparative study on the promotion effect of $\mathrm{Mn}$ and $\mathrm{Zr}$ on the stability of $\mathrm{Ni} / \mathrm{SiO}_{2}$ catalyst for $\mathrm{CO}_{2}$ reforming of methane. International Journal of Hydrogen Energy. v. 38, p. 72687279, 2013.

ZANGANEH, Rasoul; REZAEI, Mehran; ZAMANIYAN, Akbar. Dry reforming of methane to synthesis gas on $\mathrm{NiOeMgO}$ nanocrystalline solid solution catalysts. International Journal of Hydrogen Energy. v. 38, p. 3012-3018, 2013. 